\title{
Comparative Study of Bayesian and Energy Detection Including MRC Under Fading Environment in Collaborative Cognitive Radio Network
}

\author{
Shakila Zaman \\ Institute of Information Technology \\ Jahangirnagar University \\ Savar, Bangladesh
}

\author{
Risala Tasin Khan \\ Institute of Information Technology \\ Jahangirnagar University \\ Savar, Bangladesh
}

\author{
Md. Imdadul Islam \\ Computer Science and Engineering \\ Jahangirnagar University \\ Savar, Bangladesh
}

\begin{abstract}
The most important component of Cognitive Radio Network (CRN) is to sense the underutilised spectrum efficiently in fading environment for incorporating the increasing demand of wireless applications. The result of spectrum sensing can be affected by incorrect detection of the existence of Primary User (PU). In this paper, we have considered Collaborative spectrum sensing to maximise the spectrum utilisation of Cognitive Radio (CR) user. We proposed a new architecture and algorithm that shows the step by step spectrum sensing procedure using Energy detection and Bayesian detection in collaborative environment for an optimal number of users. This algorithm also includes Maximal Ratio Combining (MRC) diversity techniques in fusion centre to make a final decision under fading condition. The simulation result shows the significant optimisation of detection performance with less misdetection for large number of users. It is also observed that MRC produces better results in collaborative manner under Nakagami- $m$, Rayleigh and Normal fading. Finally in this paper, we have analysed the relative performance of different wireless channels for various SNR levels and from that analysis it concludes that ED technique works better in high SNR and BD technique works for low SNR.
\end{abstract}

Keywords-Maximal Ratio Combining; Collaborative spectrum sensing, Fading and Shadowing; Data fusion centre; Receiver operating characteristics; False alarm rate

\section{INTRODUCTION}

Modern and advanced wireless communication services are becoming scarce resources because of high data rate devices, which communicate by using electromagnetic waves. Due to fixed spectrum, nowadays this is a hard job to provide efficient bandwidth for the increasing demand [1]. Cognitive Radio Network (CRN) has become as a solution of limited spectrum problem by providing dynamic spectrum access with increasing number of users in current and future wireless communication [2]. In CRN, licensed users are known as Primary User (PU) and unlicensed users are known as Secondary User (SU) where SU's are responsible to sense the occupied spectrum and use it without any interruption by giving highest priority to PU [3]. This electromagnetic wave media is highly disposed to noise and it is tough to detect the exact transmitted signal. In presence of noise, miss detection may occur at SU. In case of misdetection, SU's senses the existence of signal power but in reality it may be just noise, or SU senses no primary signal in transmitting mode but in reality it is. So the presentation of CRN based on how finely and reliably a SU detects the unused spectrum and utilise it by $\mathrm{CR}$ users without interferences.

Spectrum detection can be done by using different techniques like, Neyman-Pearson Detection (NPD), Matched Filter (MF), Cyclostationary Detection, Energy Detection (ED) and Bayesian Detection (BD) etc. [4]-[7]. In [8], Matched Filter also known as coherent detection which can improve sensing performance by requiring less observation time and samples. Sensing of MF depends on prior knowledge about PU like modulation technique, packet structure and carrier synchronisation and timing devices of CR that is complicated to implement [9], [10]. In [11], Cyclostationary detection technique is used for detecting cyclostationary feature of PU signal. It also requires partial knowledge of PU and can easily distinguish transmitted signal from noise. This technique requires complex calculation, which is studied in [9].ED is the simplest way for sensing unknown deterministic primary signal with low complexity. It also refers as non-coherent detection, which can be implemented in both frequency and time domain that need no prior knowledge of PU [12]. BD is used to reduce the misdetection probability for a given large false alarm rated by incorporating likelihood ratio test which works better in low SNR than ED [13 ].

In this paper, we have considered the energy detection and Bayesian detection to optimise the efficient sensing in cooperative environment and to optimise total error rate. In real life, it is very challenging to estimate correct movement of PU and sense the hidden terminal independently due to fading or different obstacles like building, tree, tower etc. with high saturation loss. Collaborative Spectrum Sensing (CSS) is an intelligent and smart approach for combating multipath fading and shadowing with optimum numbers of SU [14]-[17]. In CSS, all CR users perform local measurement independently about existence or not existence of PU to make a binary decision and then forward the decision to a central Data Fusion Centre (DFC). DFC combines those decisions and makes a final decision [18]. Different conventional diversity techniques are used to combine the independent decision which are discussed in [19] and [20]. In this paper, we have considered Maximal Ratio Combining (MRC) scheme with Energy and Bayesian detection. When MRC is used, channel state information of PU is needed in DFC with a normalised weight and then is added by linear combiner. 
This paper has improved the work of [21]. This work demonstrates a clear comparison between local and collaborative sensing and has proposed a new scheme of MRC with ED to maximise spectrum detection within hidden terminal in collaborative environment. In wireless communication, fading is natural due to multipath propagation and shadowing. So, researchers are focused on detection performance over different fading channels [22], [23]. Performance of ED over Nakagami- $m$ and Rician fading is discussed in [22]. The aim of this paper is to optimise the collaborative spectrum sensing by considering ED and BD over different fading channels like Rayleigh, Nakagami- $m$ and Normal or Gaussian fading under MRC technique. It also analyses the performance of BD and ED based on SNR label.

The remainder of this work is structured as: Section 2 presents the structure of a signal model and mathematical formulation about $\mathrm{ED}$ and $\mathrm{BD}$ in local sensing. In Section 2, different fading channels characteristics like Nakagami- $m$, Rayleigh and Gaussian with tradition MRC is formulated with a new face. A complete algorithm, corresponding flowchart and proposed architecture of our collaborative spectrum sensing system are also deliberated in this section. Section 3 discusses about the simulation results with required parameter and gives an analysis of access opportunity of collaborative CRN. Final conclusion is given in Section 4.

\section{SIGNAL MODEL}

\section{A. Signal model of local spectrum sensing}

The main goal of spectrum sensing is to increase efficient use of spectrum hole and monitor the channel continuously to provide primary user precedence. In this paper, two most popular detection techniques is used like Energy detection and Bayesian detection to maximise the accessibility in an occupied channel based on SNR estimation. For binary signal detection two hypothesis are chosen to specify a decision rule about the presence or absence of PU that is referred as statistical decision. By following the term of signal the detection problem is solved using following hypothesis function [16],

$$
\phi(x)= \begin{cases}x: \phi(x)=0 ; & H_{0} \\ x: \phi(x)=1 ; & H_{1}\end{cases}
$$

Where $H_{0}$ denotes as Null hypothesis that indicate there is no signal without noise, $H_{1}$ indicate that primary user is in operation mode that produce the result of presence of primary user.

In cognitive radio network, we consider $\mathrm{N}$ number of secondary users for spectrum sensing and each user senses the spectrum hole independently. For $i^{\text {th }}$ secondary user that is independently and identically distributed [24], local spectrum sensing is determined by following the signal model including two hypothesis [21],

$$
y_{i}[k]= \begin{cases}w_{i}[k] ; & H_{0} \\ \delta_{i} e^{j \theta_{i} x[k]+w_{i}[k] ;} & H_{1}\end{cases}
$$

Where $y_{i}[k]$ is denotes as received signal for $x[k]$ primary user's transmitted signal at $\mathrm{i}^{\text {th }}$ secondary user and $x[k]$ follows the Gaussian random process with zero mean and variance $\zeta_{n}^{2}$. At the signal detector the sample sequence set of secondary users refers as $\mathrm{i} \epsilon\{1,2,3, \ldots, \mathrm{N}\}, w_{i}[k]$ is additive noise that produce null hypothesis and indicate that there is no primary user. $\delta_{i} e^{j \theta_{i}}$ is the complex factor of channel gain between transmitter and receiver. The term $\delta_{i} e^{j \theta_{i}} x[k]+$ $w_{i}[k]$ indicates that primary user is detected with $H_{1}$ hypothesis.

\section{B. Local spectrum sensing using Energy detection}

Energy detection is also known as non-coherent detection that can detect the signal energy by ignoring the structure of the signal. In case of unknown feature of a signal, energy detection could make better result. In Figure 1, energy detection technique collects transmitted signal bandwidth in specified sensing interval $t_{i}$. Received Sampling signals are prefilled using Bandpass filter and then square them using magnitude squaring device. Squared signals are integrated with respect to specified time interval to measure the test statistics.

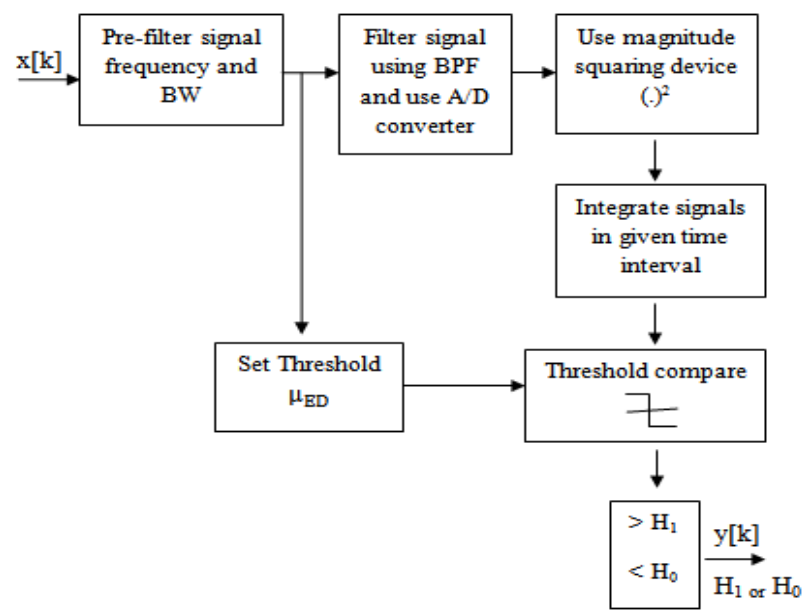

Fig. 1. Spectrum sensing using Energy detection

In this energy detection, process test hypothesis is compared with predefined threshold value $\mu_{E D}$, which is measured based on signal noise, energy and sampling size. Functionally test statistics is given by [25],

$$
V(y)=\frac{1}{M} \sum_{i=0}^{M} y_{i}[k]^{2}
$$

where,

$V(y)=$ test statistics,

$\mathrm{M}=$ sampling size of received signal.

In this case, an efficient decision rule is introduced by comparing predefined threshold with test statistics where received signal vector is $y=\left\{y_{1}[k], y_{2}[k], y_{3}[k], \ldots \ldots, y_{N}[k]\right\}$ that varies only two random variable set $\{0,1\}$ that produce the hypothesis $H_{j}(j=0,1)$. Formally the decision rule is given by,

$$
\begin{aligned}
& H_{0} \ldots \ldots \ldots \ldots . . . \text { if } \mu_{E D, i}<V(y) \\
& H_{1} \ldots \ldots \ldots \ldots . . . \text { if } \mu_{E D, i}>V(y)
\end{aligned}
$$

where,

$$
\mu_{E D, i}=\left|\xi_{x} y_{i}[k]\right|^{2} \text {, }
$$


$\xi_{\mathrm{x}}=$ Power budget at Primary user.

To determine the efficient measurement of test statistics it is very important to identify the number of sample and threshold value, that are calculated based on two important detection probability parameters $P_{d}$ and $P_{f a} . P_{d}$ is denoted as probability of detection and $P_{f a}$ is denoted as probability of false alarm. Threshold and efficient sample size is measured by given equations [25],

$$
\begin{gathered}
M=\frac{2\left(Q^{-1}\left(P_{f a}\right)-Q^{-1}\left(P_{d}\right)\right)^{2}}{\gamma_{i}{ }^{2}} \\
\left.\mu_{\mathrm{ED}}=\sqrt{2 M} Q^{-1}\left(P_{f a}\right)+M\right) \zeta_{n}^{2}
\end{gathered}
$$

where,

$\gamma_{i}=\mathrm{SNR}$ for ith SU,

$\gamma_{i}=\xi_{\mathrm{s}}\left|\delta_{i} e^{j \theta_{i}}\right|^{2} / \mathrm{N}_{0}$

$\mathrm{N}_{0}=$ One sided power spectral density.

Since PU is surrounded by different fading and obstruction, it is very tough to make a correct spectrum sensing decision with respect to $\left(P_{f a}\right)$ and $\left(P_{m d}\right)$. A correct dicision matrix is given in Table 1 according to Figure 2 that produces the result about existence or not existence of PU.

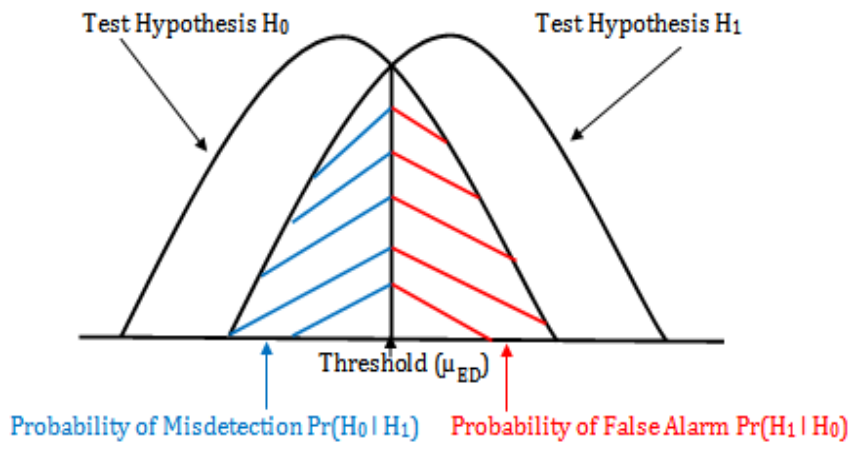

Fig. 2. Block diagram of detection hypothesis

TABLE I. CORRECT DECISION MATRIX

\begin{tabular}{|l|l|l|}
\hline & \multicolumn{2}{|l|}{ Decision rules } \\
\hline Final decision & \multicolumn{1}{|c|}{$\boldsymbol{H}_{\mathbf{1}}$} & \multicolumn{1}{c|}{$\boldsymbol{H}_{\mathbf{0}}$} \\
\hline Primary user is & Detection Probability & $\begin{array}{l}\text { Misdetection probability } \\
\text { present }\left(H_{1}\right)\end{array}$ \\
\hline $\begin{array}{l}\text { Primary user is } \\
\text { absent }\left(H_{0}\right)\end{array}$ & False alarm & $\left(P_{m d}\right)$ \\
\hline
\end{tabular}

For a non-fading environment the statistical measurement of detection probability is given as [23],

$$
\begin{aligned}
P_{d, i}\left(M, \mu_{\mathrm{ED}, \mathrm{i}}\right) & =\operatorname{Pr}\left(\text { Primaryuserispresent } \mid H_{1}\right) \\
& =\operatorname{Pr}\left(H_{1} \mid H_{1}\right) \\
& =\operatorname{Pr}\left(V(y)>\mu_{\mathrm{ED}} \mid H_{1}\right) \\
& =Q_{u}\left(\sqrt{2 \gamma_{i}}, \sqrt{\mu_{\mathrm{ED}, \mathrm{i}}}\right)
\end{aligned}
$$

where,

$\mathrm{u}=$ the time-bandwidth product $=\mathrm{TW}$

$\mu_{\mathrm{ED}, \mathrm{i}}$ threshold value for ith secondary user,
$Q_{u}(p, q)$ is the generalised Marcum-Q function which is formulated as [23],

$$
Q_{u}(p . q)=\frac{1}{p^{u-1}} \int_{q}^{\infty} t^{u} e^{-\frac{t^{2}+p^{2}}{2}} I_{u-1}(p t) d t
$$

And $I_{u-1}(p)$ is modified Bessel function of the u-1 order. Therefor using this function the probability of detection for the $\mathrm{i}^{\text {th }}$ user can be written as,

$$
P_{d, i}\left(M, \mu_{\mathrm{ED}, \mathrm{i}}\right)=\frac{1}{{\sqrt{2 \gamma_{i}}}^{u-1}} \int_{\sqrt{\mu_{\mathrm{ED}, \mathrm{i}}}}^{\infty} t^{u} e^{-t^{2}+{\sqrt{2 \gamma_{i}}}^{2}} I_{u-1}\left(\sqrt{2 \gamma_{i}} t\right) d t
$$

Since integral calculation of detection probability makes high complexity we can represent the formula as series function of Marcum-Q function,

$$
\begin{aligned}
P_{d, i}\left(M, \mu_{\mathrm{ED}, \mathrm{i}}\right)= & e^{-\frac{\mu_{\mathrm{ED}, \mathrm{i}}}{2}} \sum_{a=0}^{u-1} \frac{\left(\frac{u}{2}\right)^{a}}{a !} \\
& +e^{-\frac{\mu_{\mathrm{ED}, \mathrm{i}}}{2}} \sum_{b=0}^{u} \frac{\left(\frac{u}{2}\right)^{b}}{b !}\left(1-e^{-\gamma_{i}} \sum_{c=0}^{a-u} \frac{\gamma_{i}^{c}}{c !}\right)
\end{aligned}
$$

Therefore, Probability of misdetection is given as,

$$
\begin{aligned}
P_{m d, i}\left(M, \mu_{\mathrm{ED}, \mathrm{i}}\right) & =\operatorname{Pr}\left(\text { Primary user is absent } \mid H_{1}\right. \\
& =\operatorname{Pr}\left(H_{0} \mid H_{1}\right) \\
& =1-P_{d, i}\left(M, \mu_{\mathrm{ED}, \mathrm{i}}\right)
\end{aligned}
$$

And statistical calculation of false alarm is written as,

$$
\begin{aligned}
P_{f a, i}\left(M, \mu_{\mathrm{ED}, \mathrm{i}}\right) & =\operatorname{Pr}\left(\text { Primary user is present } \mid H_{0}\right. \\
& =\operatorname{Pr}\left(H_{1} \mid H_{0}\right) \\
& =\operatorname{Pr}\left(V(y)>\mu_{\mathrm{ED}} \mid H_{0}\right) \\
& =\frac{\Gamma\left(u, \mu_{\mathrm{ED}, \mathrm{i}} / 2\right)}{\Gamma(u)}
\end{aligned}
$$

\section{Local spectrum sensing using Bayesian Detection}

Bayesian detection method is used in a prior statistics of $\mathrm{PU}$ movement and signalling information of PU to improve the throughput of SU sensing to utilise the unused spectrum. Bayesian detector works as a likelihood ratio test detector, which can make better performance in low and high SNR in binary hypothesis testing. Decision of the testing will produce by comparing this likelihood ratio with predefined threshold which is shown in Figure 3.

The main goal of Bayesian detector is to reduce the cost or risk for making the incorrect decision. Expected minimise cost expression is defined as,

$$
C=C_{1 / 0} P\left(C_{1 / 0}\right)+C_{0 / 1} P\left(C_{0 / 1}\right)+C_{1 / 1} P\left(C_{1 / 1}\right)+C_{0 / 0} P\left(C_{0 / 0}\right)
$$

where, $C_{a b}(a=0,1$ and $b=0,1)$ is the estimation of the cost that can make a detection statistics with binary hypothesis test. According to decision rule a clear cost matrix with detection probability is given in Table 2 . 
TABLE II. COST MATRIX WITH BINARY HyPOTHESIS

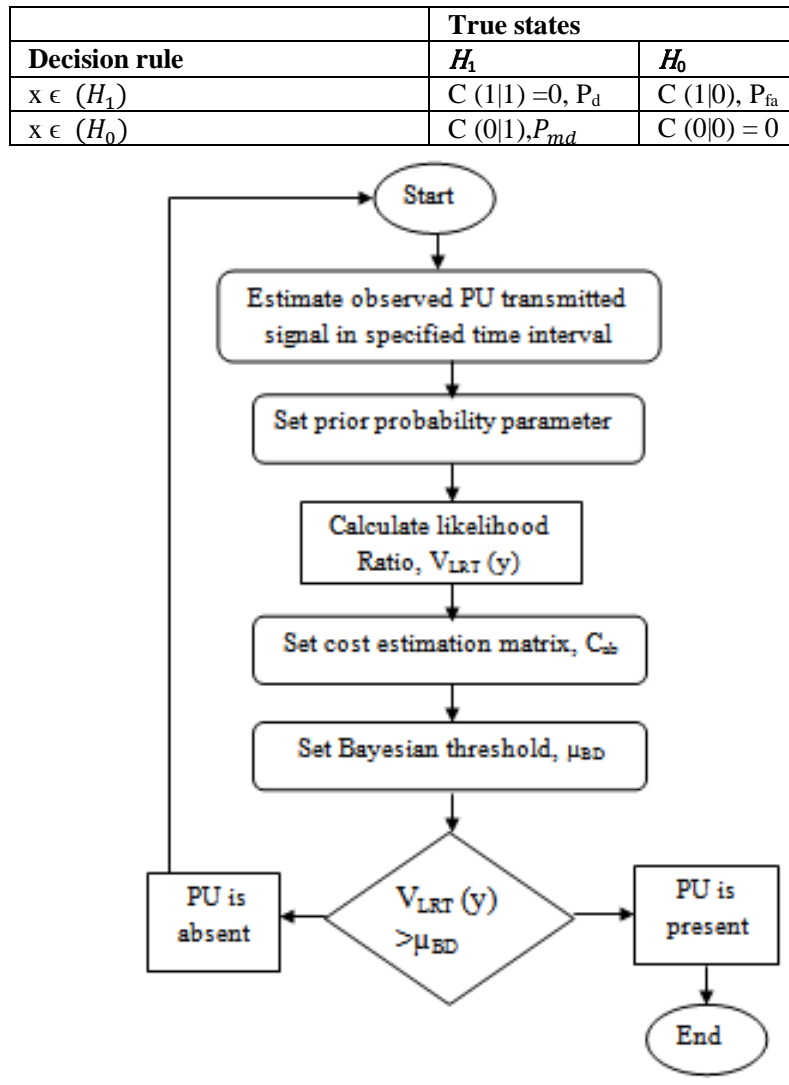

Fig. 3. Process flow diagram of Bayesian detection

For the two hypothesis testing with prior information, the likelihood ratio test is formulated as [26],

$$
\begin{aligned}
V_{L R T}\left(y_{i}\right)= & \frac{P\left(y_{i} \mid H_{1}\right)}{P\left(y_{i} \mid H_{0}\right)}=\frac{P\left(H_{1} \mid y_{i}\right) P\left(y_{i}\right)}{P\left(H_{1}\right)} \times \frac{P\left(H_{0}\right)}{P\left(H_{0} \mid y_{i}\right) P\left(y_{i}\right)} \\
& =\frac{P\left(H_{1} \mid y_{i}\right) P\left(H_{0}\right)}{P\left(H_{0} \mid y_{i}\right) P\left(H_{1}\right)}
\end{aligned}
$$

Then the likelihood ratio is compared with threshold of Bayesian detection that is suitable to derive optimal detector,

$$
\begin{gathered}
V_{L R T}(y)^{H_{1}} \stackrel{H}{H}_{H_{0}} \\
\mu_{B D}
\end{gathered}
$$

Where $\mu_{B D}=\frac{P\left(H_{0}\right)\left(C_{1 \mid 0}-C_{0 \mid 0}\right)}{P\left(H_{1}\right)\left(C_{0 \mid 1}-C_{1 \mid 1}\right)}$

Bayesian detector is used to minimise the Bayesian cost to maximise spectrum utilisation. This function is related to false alarm probability and correct decision probability,

$$
\operatorname{Max} P\left(H_{0}\right)\left(1-P_{f a}\right)+P\left(H_{1}\right) P_{d}
$$

\section{Spectrum sensing under fading channel}

In wireless communication system, fading occurs due to multipath propagation and shadowing. Measurement of detection performance of energy detection and Bayesian detection over fading channel is very important to meet the spectrum sensing challenges to improve transmission performance. Probability of detection in fading condition is measured using following equation,

$$
\begin{aligned}
& \overline{P_{d}}=\int_{0}^{\infty} P_{d}(\gamma, \mu) f(\gamma, \bar{\gamma}) d \gamma \\
&=\int_{0}^{\infty} Q_{u}(\sqrt{2 \gamma}, \sqrt{\mu}) f(\gamma) d \gamma
\end{aligned}
$$

where, $f(\gamma, \bar{\gamma})$ is refers as probability density function for different fading channels.

In case of Nakagami- $m$ fading channel, the probability density function is given by,

$$
f(\gamma, \bar{\gamma})=\frac{m^{m}(\gamma)^{m-1}}{(\bar{\gamma})^{m} \Gamma(m)} e^{-\frac{m \gamma}{\bar{\gamma}}}
$$

Therefore, average probability of detection over Nakagami$m$ fading by following equation (7) is formulated as [23],

$\overline{P_{d}}=e^{-\frac{\mu}{2}} \sum_{a=0}^{u-1} \frac{\left(\frac{u}{2}\right)^{a}}{a !}+e^{-\frac{\mu}{2}} \sum_{b=0}^{u} \frac{\left(\frac{u}{2}\right)^{b}}{b !}$

$\left(1-\frac{m^{m}}{(\bar{\gamma})^{m} \Gamma(m)} \sum_{c=0}^{b-u} \frac{\int_{0}^{\infty}(\gamma)^{c+m-1} e^{-\frac{m+\overline{\bar{\gamma}}}{\bar{\gamma}}} d \gamma}{c !}\right)$

$=e^{-\frac{\mu}{2}} \sum_{a=0}^{u-1} \frac{\left(\frac{u}{2}\right)^{a}}{a !}+e^{-\frac{\mu}{2}} \sum_{b=0}^{u} \frac{\left(\frac{u}{2}\right)^{b}}{b !}$

$\left(1-\left(\frac{m}{\bar{\gamma}+m}\right)^{m} \sum_{c=0}^{b-u} \frac{(m+c-1) !}{\Gamma(m) c !}\left(\frac{\bar{\gamma}}{\bar{\gamma}+m}\right)^{c}\right)$

where, $m$ is shape parameter of Nakagami- $m$ channel

The probability density function for Rayleigh fading channel is,

$$
f(\gamma, \sigma)=\frac{\gamma}{\sigma^{2}} e^{-\frac{\gamma^{2}}{2 \sigma^{2}}}
$$

where, $\boldsymbol{\sigma}$ is scale parameter of Rayleigh distribution

And in case of Normal or Gaussian fading channel, the probability density function is,

$$
f(\beta, \partial, x)=\frac{1}{\partial \sqrt{2 \pi}} e^{-\frac{(x-\beta)^{2}}{2 \partial^{2}}}
$$

where, $\beta$ is the expectation of the distribution and $\partial^{2}$ is the variance of the normal distribution

We can calculate the spectrum detection probability over Rayleigh and Normal fading channel to apply the corresponding PDF equation (16) and (17) in equation (7). 


\section{E. Sensing under fading channels in collaborative environment including $M R C$}

a) Formulation: For collaborative spectrum environment, we have considered $\mathrm{N}$ number of secondary users to sense the occupied spectrum to get an efficient result. In collaborative CRN, $\mathrm{N}$ number of $\mathrm{SU}$ senses the spectrum individually in a specified time interval to detect the real state of PU. Figure 4, determine PU activity with occupied states $D$ $=\left\{d_{1}, d_{2}, \ldots \ldots \ldots, d_{T}\right\}$, for specified time interval states $t=\{1$, $2, \ldots \ldots \ldots, T\}$. But, for the hidden spectrum hole SU generates its observation sequence $O=\left\{o_{1}, o_{2}, \ldots \ldots \ldots, o_{T}\right\}$, based on their local detection procedure. This observation set represent sensing information about the existence of not existence of PU in transmission mode. All SU transmit their observation information to DFC using local sensing method. Then DFC makes the final decision whether the SU finally transmit or not.

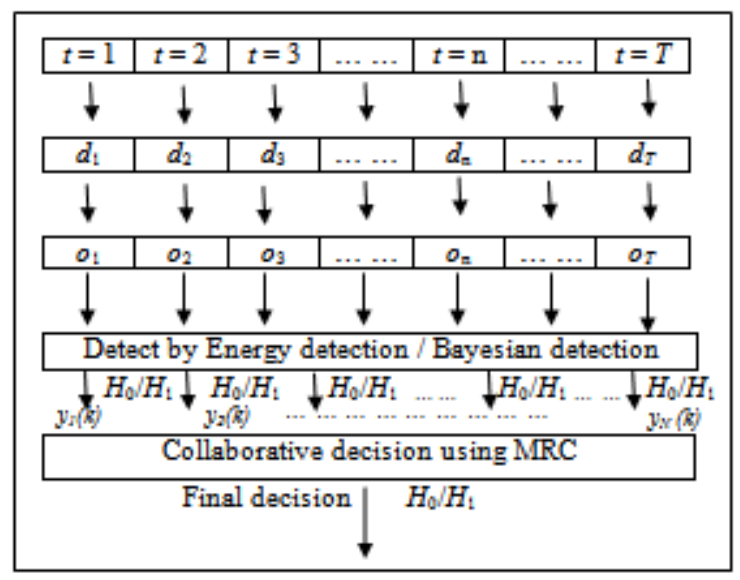

Fig. 4. Block diagram of proposed Collaborative detection using MRC

For $\mathrm{N}$ number of collaborative user where $\mathrm{N}=1,2,3, \ldots, \mathrm{N}_{\mathrm{m}}$, the probability of detection in DFC is written as [21],

$$
\begin{gathered}
\Phi_{d}=1-\left(1-P_{d}\right)^{N} \\
\varpi_{d}=1-\left(1-Q_{u}(\sqrt{2 \gamma}, \sqrt{\mu})\right)^{N}
\end{gathered}
$$

The probability of false alarm for collaborative detection is written as,

$$
\begin{gathered}
\Phi_{f a}=1-\left(1-P_{f a}\right)^{N} \\
\widetilde{\varpi}_{f a}=1-\left(1-\frac{\Gamma(u, \mu / 2)}{\Gamma(u)}\right)^{N}
\end{gathered}
$$

To make an effective detection result DFC use different diversity method to combine the given sensing form all SU. One of the most popular diversity schemes is maximum ratio combining scheme. In this work, we proposed a collaborative environment with MRC diversity in different fading channels. The DFC collects all information and combine them using linear combiner. In MRC diversity, the complex envelop of received signal for $i^{t h}$ individual branches of SU is formulated as [27],

$$
\begin{gathered}
\tilde{\mathrm{z}}[k]=\sum_{\substack{i=1 \\
N_{m}}}^{\xi_{i} \tilde{y}_{i}[k]} \\
=\sum_{i=1}^{N_{m}} \xi_{i}\left[\delta_{i} e^{\left.j \theta_{i} x[k]+\widetilde{w}_{i}[k]\right]}\right.
\end{gathered}
$$

where,

$\delta_{i} e^{j \theta_{i}}=$ composite channel achievement in fading, $\xi_{i}=$ weighted factor for each channel, $\sum_{i=1}^{N_{m}} \xi_{i}\left[\delta_{i} e^{j \theta_{i}} x[k]=\right.$ compound envelop of received signal, $\sum_{i=1}^{N_{m}} \xi_{i} \widetilde{w}_{i}[k]=$ complex envelop of received noise.

Then the detection hypothesis is expressed as,

$$
\tilde{z}[k]= \begin{cases}\sum_{i=1}^{N_{m}} \xi_{i} \tilde{y}_{i}[k] ; & H_{0} \\ \sum_{i=1}^{N_{m}} \xi_{i}\left[\delta_{i} e^{\left.j \theta_{i} x[k]+\widetilde{w}_{i}[k]\right] ;}\right. & H_{1}\end{cases}
$$

To maximise the detection statistics in collaborative sensing, MRC technique introduce an instantaneous SNR that is indicated as $\gamma_{M R C}$. In DFC, this is calculated by summarising given all individual secondary users SNR using linear combiner. That is,

$$
\gamma_{M R C}=\sum_{i=1}^{N_{m}} y_{i}
$$

Therefore, we can write the detection probability under MRC method as,

$$
\begin{gathered}
P_{d, M R C}=e^{-\frac{\mu_{\mathrm{ED}}}{2}} \sum_{a=0}^{u-1} \frac{\left(\frac{u}{2}\right)^{a}}{a !}+e^{-\frac{\mu_{\mathrm{ED}}}{2}} \sum_{b=0}^{u} \frac{\left(\frac{u}{2}\right)^{b}}{b !}(1- \\
e^{\left.-\gamma_{M R C} \sum_{c=0}^{a-u} \frac{\gamma_{M R C}{ }^{c}}{c !}\right)}
\end{gathered}
$$

This equation will used to express the detection probability over Nakagami- $m$, Rayleigh and Normal fading channels by using equation (15). For Nakagami- $m$ fading the detection probability under MRC is mathematically calculated as,

$$
\begin{gathered}
\overline{P_{d, M R C}}=e^{-\frac{\mu}{2}} \sum_{a=0}^{u-1} \frac{\left(\frac{u}{2}\right)^{a}}{a !}+e^{-\frac{\mu}{2}} \sum_{b=0}^{u} \frac{\left(\frac{u}{2}\right)^{b}}{b !}(1- \\
\left.\left(\frac{m}{\overline{\gamma_{M R C}}+m}\right)^{m} \sum_{c=0}^{b-u} \frac{(m+c-1) !}{\Gamma(m) c !}\left(\frac{\overline{\gamma_{M R C}}}{\overline{\gamma_{M R C}}+m}\right)^{c}\right)
\end{gathered}
$$

So, collaborative detection and false alarm probability under MRC can be expressed by,

$$
\rrbracket_{d, M R C}=1-\left(1-\overline{P_{d, M R C}}\right)^{N}
$$

And

$$
\mathrm{\sigma}_{f a}=1-\left(1-P_{f a}\right)^{N}
$$

b) System Algorithm:

To understand the working procedure of our system a wellorganised and smart algorithm is introduced. This algorithm shows the step by step spectrum sensing procedure using BD and $\mathrm{ED}$ in collaborative environment for an optimal number of users. This algorithm also includes MRC diversity technique in DFC to make a final decision (Algorithms 1 to 3). 


\section{Algorithm 1 Steps to estimate Collaborative Spectrum}

Sensing under different fading channels using MRC

\section{Initial Step}

Step 1: Cognitive Radio user received transmitted signal independently trough specified sensing period with $N=1,2$, $3, \ldots \ldots, N_{m}$ no-cooperative SU.

Local sensing

Step 2: For N individual users.

Step 3: Select the received signal and filter the signal locally at each user.

Step 4: Perform PU detection method (Energy detection or Bayesian detection).

Step 5: Take independent decision using local ED or BD based on SNR level using algorithm 2 and 3.

Step 6: Report independent sensing decision $\mathrm{H}_{j}$ to DFC.

Step 7: End For.

Final decision in DFC

Step 6: DFC produces the final result using MRC under

Nakagami- $m$, Rayleigh and Normal Fading channels.

Step 7: Estimate $\xi_{i}$ and $\delta_{i} e^{j \theta_{i}}$ for independent user for equation (20)

Step 8: Compute $\gamma_{M R C}$.

Step 10: Calculate $V(y)$.

Step 12: Match $V(y)$ with $\mu_{\mathrm{BD}}$ or $\mu_{\mathrm{ED}}$.

Step 13: If $V(y)$ is greater than threshold value then DFC makes $\mathrm{H}_{1}$ as a final result else produce $\mathrm{H}_{0}$.

Step 13: Compute $P_{d}, P_{f a}$ and $P_{m d}$ under different fading channel using MRC.

Step 14: Calculate $\mathbb{\varpi}_{d}$ and $\mathbb{\Phi}_{f a}$ to evaluate Collaborative sensing proficiency under fading channels using MRC.

Algorithm 2 Steps to calculate local sensing using Energy detection

Step 1: SU takes the received signal and pass through BPF

Step 2: Estimate Power Spectral Density (PSD).

Step 3: Integrate PSD and determine fixed threshold $\mu_{\mathrm{ED}}$ using parameters.

Step 4: Compute test statistics $V\left(y_{i}\right)$.

Step 5: Compare $V\left(y_{i}\right)$ and $\mu_{\mathrm{ED}}$, for $V\left(y_{i}\right)>\mu_{\mathrm{ED}, \mathrm{i}}$ produce $\mathrm{H}_{1}$ otherwise produce $\mathrm{H}_{0}$.

\section{Algorithm 3 Steps to calculate local sensing using Bayesian} detection

Step 1: SU takes the received signal at specified sensing time.

Step 2: Set prior probability parameters.

Step 4: Compute likelihood ratio $V_{L R T}\left(y_{i}\right)$.

Step 5: Set cost estimation matrix.

Step 2: Calculate posterior.

Step 4: Calculate $\mu_{\mathrm{BD}, \mathrm{i}}$ using step 2, 3 and 4.

Step 5: Compare $V_{L R T}\left(y_{i}\right)$ and $\mu_{\mathrm{BD}, \mathrm{i}}$.

Step 6: if $V_{L R T}\left(y_{i}\right)>\mu_{\mathrm{BD}, \mathrm{i}}$ then produce $\mathrm{H}_{1}$ otherwise produce
$\mathrm{H}_{0}$.

\section{SIMULATION AND RESUlT}

This section is about the performance of detection in collaborative environment under different fading channels like Rayleigh, Normal and Nakagami- $m$ using MRC. In addition, we also compare the performance between Energy detection and Bayesian detection based on specified SNR.

\section{A. Simulation parameters}

To estimate the collaborative performance under fading channel using MRC, the numerical simulation parameters followed by Energy and Bayesian detection are considered in Table 3.

TABLE III. PARAMETERS FOR EVALUATE ENERGY AND BAYSIEN DETECTION UNDER DIFFERENT FADING USING MRC

\begin{tabular}{|c|c|c|}
\hline Parameter & Description & Value \\
\hline$\mu_{E D} / \mu_{B D}$ & Threshold & $0.001-0.02$ \\
\hline $\bar{\gamma}$ & Average SNR & $-30 \mathrm{db}-20 \mathrm{db}$ \\
\hline $\mathrm{T}$ & Sensing time & $40-300 \mathrm{~ms}$ \\
\hline W & Sampling bandwidth & $50-500 \mathrm{~Hz}$ \\
\hline$\sigma$ & $\begin{array}{l}\text { Scale parameter for } \\
\text { Rayleigh fading }\end{array}$ & $0.03-0.08$ \\
\hline$N$ & Number of users & $1-10$ \\
\hline$M$ & Number of samples & $50-1000$ \\
\hline$m$ & $\begin{array}{l}\text { Shape parameter for } \\
\text { Nakagami-m fading }\end{array}$ & $2-4$ \\
\hline$\partial^{2}$ & Variance for Normal fading & 0.001 \\
\hline
\end{tabular}

\section{B. Simulation Results}

In the simulation result section, Receiver Operating Characteristics (ROC) curves are used to recognise the access probability of collaborative sensing by measuring the interchange between $P_{d}$ and $P_{f a}$ against the different SNR levels. This segment delivers simulation and analytical results to verify and compare the ROC curves in sensing condition. All figures show that theoretical results are very close to simulation result. Therefore we can say that more than $95 \%$ confidence level is achieved.

Figure 5 shows the impact of collaborative detection for different numbers of users. It indicates that probability of collaborative detection will increases at a large number of users with fewer false alarms. Figure 6 demonstrates $P_{d}$ against $P_{f a}$ for various sampling rates. It has been observed for the figure that the detection probability rises with a large number of sampling.

Figure 7 shows the performance of Rayleigh fading under MRC using Bayesian detection. Though for the increased number of antenna MRC works better, but the ROC curve for collaborative with MRC produce superior detection than local sensing. Therefore using Table 4 we can say that Bayesian detection works in low SNR under Rayleigh fading where $M=$ 200. 


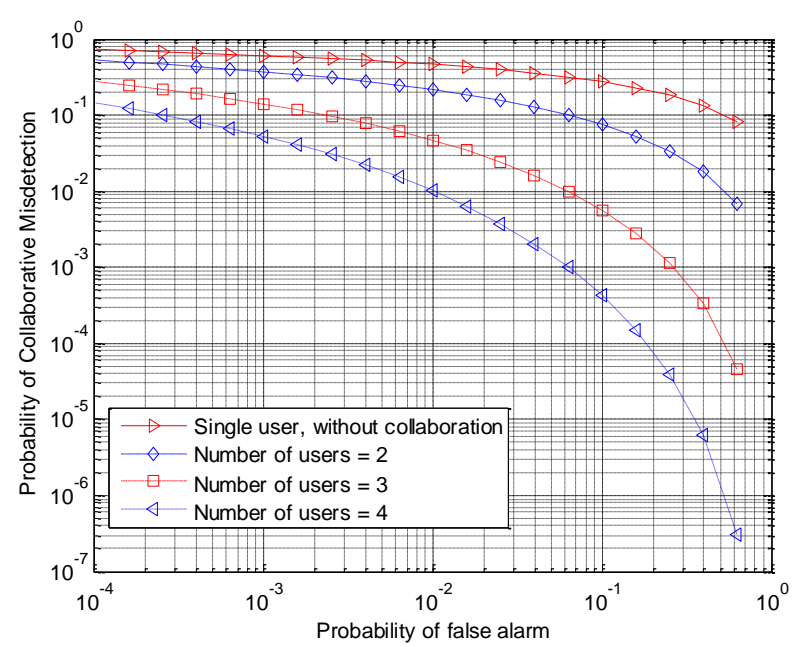

Fig. 5. Complementary ROC curves of collaborative Missdetection for different users

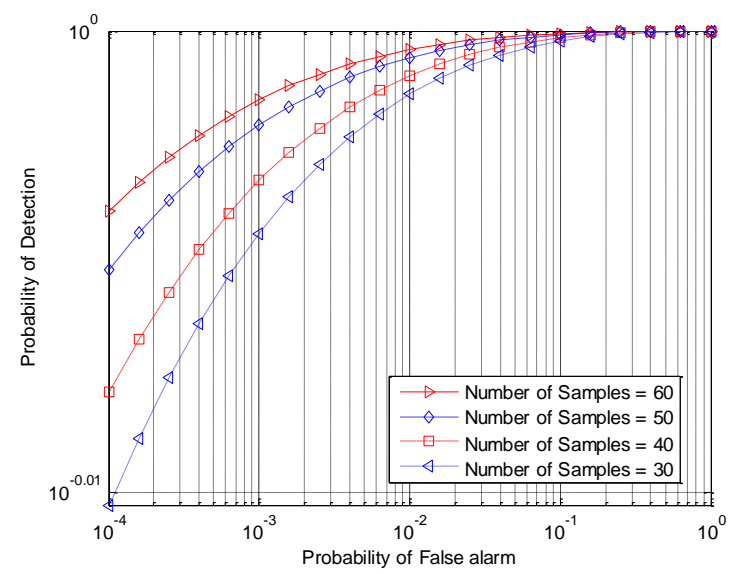

Fig. 6. Variation of the probability of detection against false alarm for various Number of sample rates

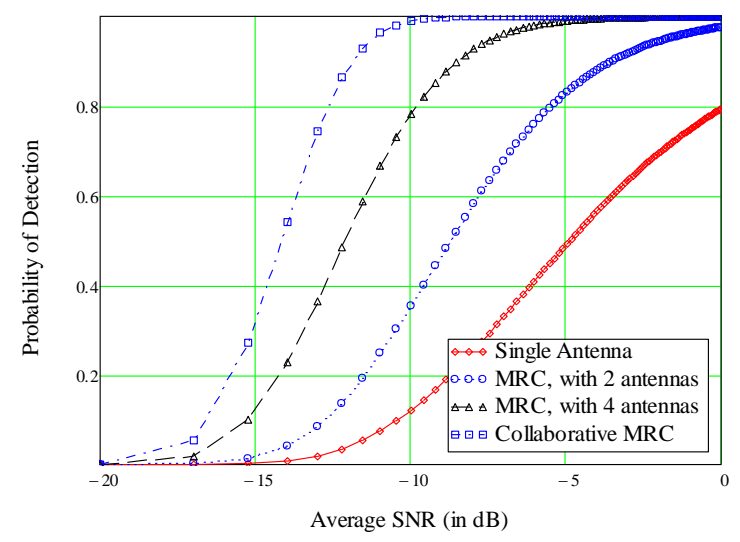

(a) Detection probability under Rayleigh fading

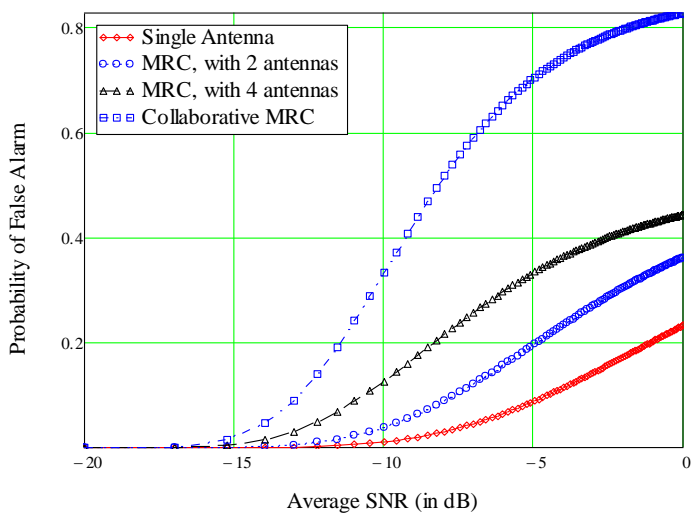

(b) False alarm probability under Rayleigh fading

Fig. 7. ROC curves for probability of detection and false alarm against average SNR for Bayesian detection under Rayleigh fading channel

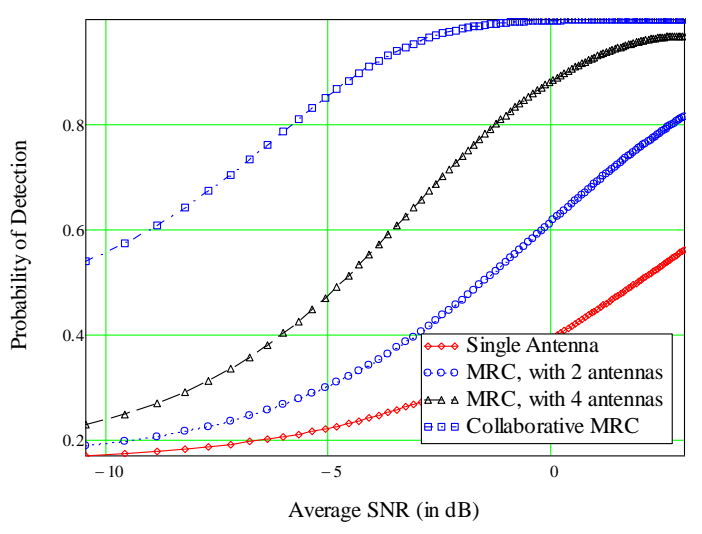

a) Detection probability under Rayleigh fading

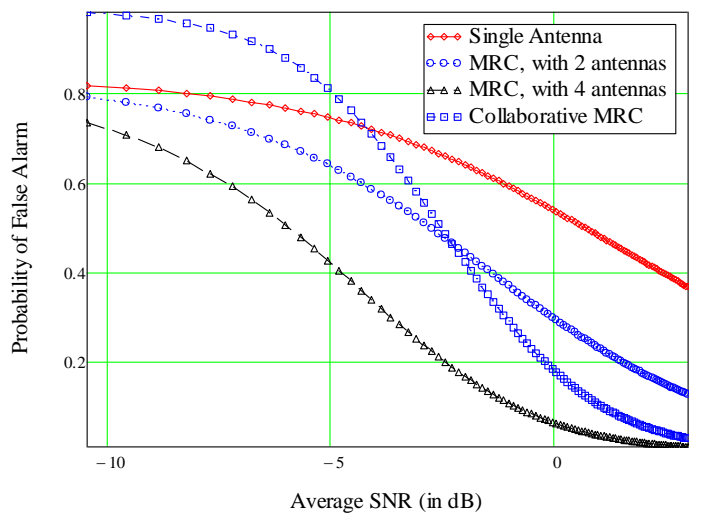

(b) False alarm probability under Rayleigh fading

Fig. 8. Detection and False alarm probability curves VS. average SNR for Energy detection under Rayleigh fading channe 


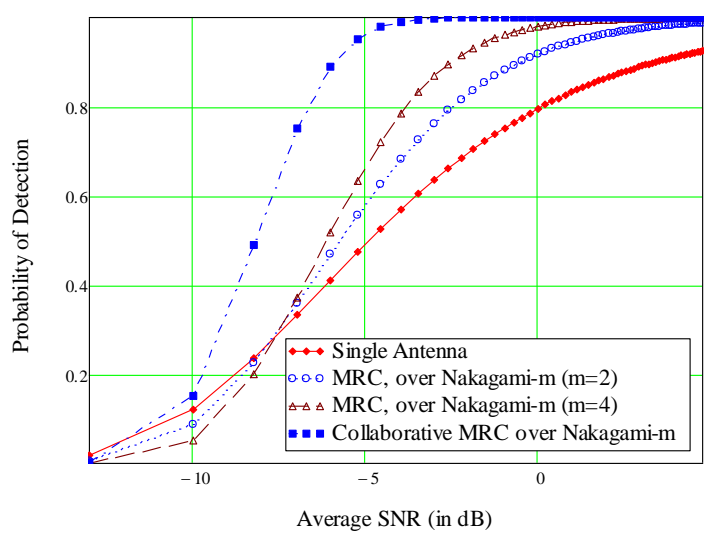

(a) Detection probability under Nakagami- $m$ fading

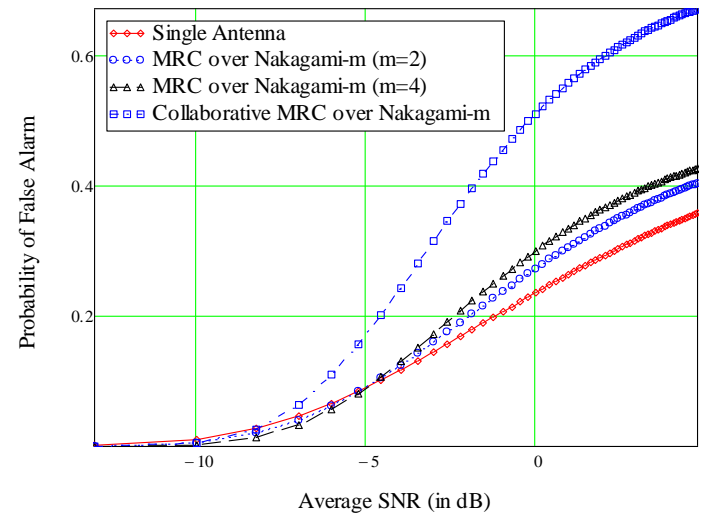

(b) False alarm probability under Nakagami- $m$

Fig. 9. Complementary ROC curves of Probability of detection and false alarm for Bayesian detection under Nakagami-m channel

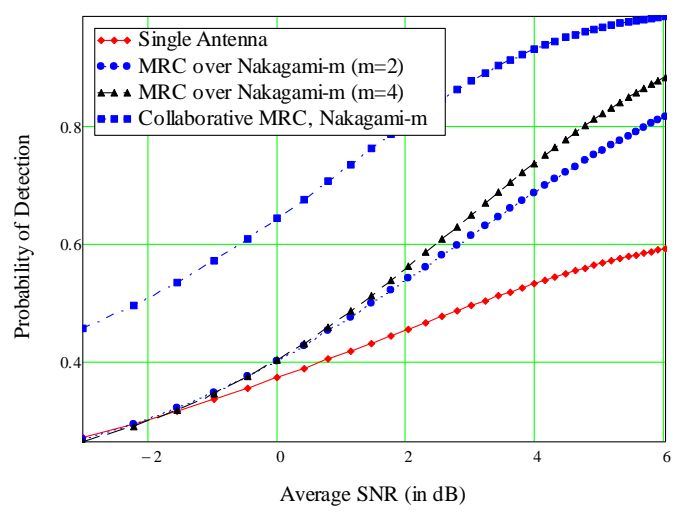

(a) Detection probability under Nakagami- $m$ fading

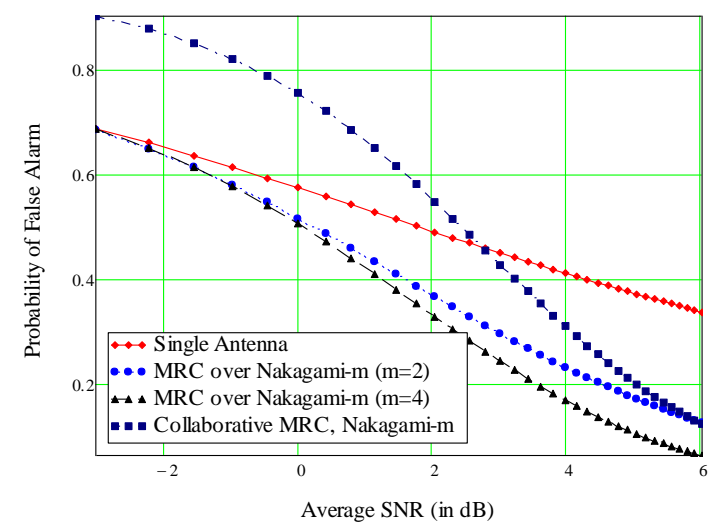

(b) False alarm probability under Nakagami- $m$ fading

Fig. 10. ROC curves for detection and false alarm probability against average SNR under Nakagami- $m$ channel using Energy detection

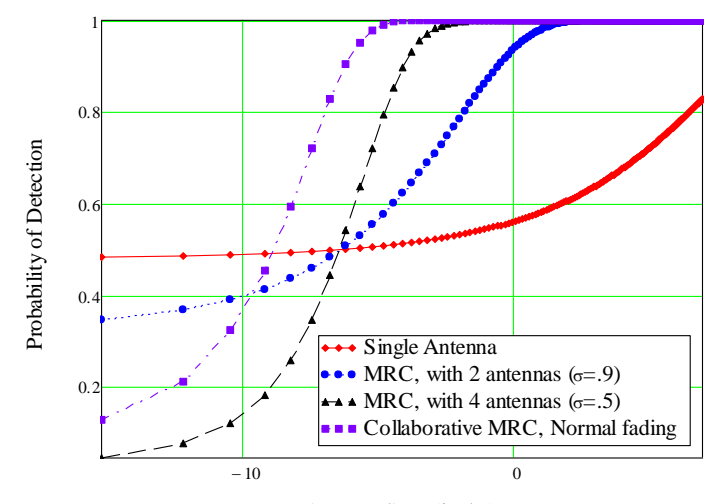

Average SNR (in dB)

(a) Detection Probability under Normal fading

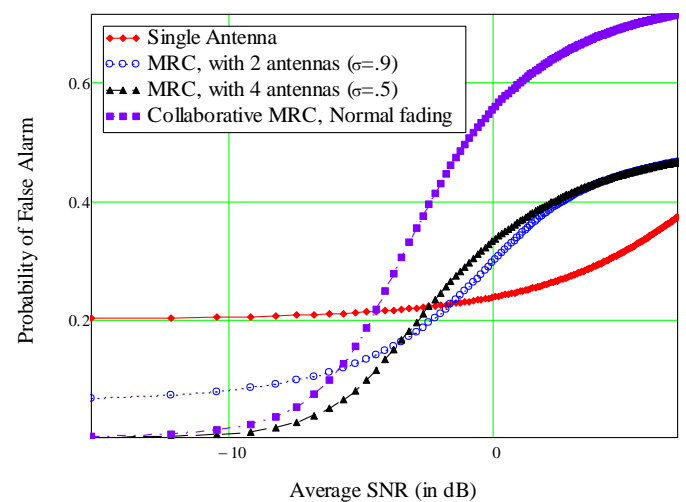

(b) False alarm probability under Normal fading

Fig. 11. Performance curves of detection and False alarm probability VS average SNR under Normal fading channel using Bayesian detection 
TABLE IV. Relative PERFormance OF WiRELESS ChanNELS

\begin{tabular}{|c|c|c|c|c|c|c|}
\hline \multirow[t]{2}{*}{$\begin{array}{l}\text { Number of Antenna with } \\
\text { MRC }\end{array}$} & \multicolumn{2}{|c|}{ Rayleigh Fading } & \multicolumn{2}{|c|}{ Nakagami- $m$ Fading } & \multicolumn{2}{|c|}{ Normal Fading } \\
\hline & $\begin{array}{l}\text { BD, } \\
\bar{\gamma}=-10 \mathrm{db}\end{array}$ & $\begin{array}{l}\text { ED, } \\
\bar{\gamma}=-\mathbf{2 d b}\end{array}$ & $\begin{array}{l}\text { BD, } \\
\bar{\gamma}=-5 \mathrm{db}\end{array}$ & $\begin{array}{l}\text { ED, } \\
\bar{\gamma}=\mathbf{4 d b}\end{array}$ & $\begin{array}{l}\text { BD, } \\
\bar{\gamma}=-5 \mathrm{db}\end{array}$ & $\begin{array}{l}\text { ED, } \\
\bar{\gamma}=4 \mathrm{db}\end{array}$ \\
\hline \multirow{2}{*}{ Single antenna } & $p_{d}=10 \%$ & $p_{d}=30 \%$ & $p_{d}=43 \%$ & $p_{d}=43 \%$ & $p_{d}=50 \%$ & $p_{d}=62 \%$ \\
\hline & $p_{f a}=2 \%$ & $p_{f a}=70 \%$ & $p_{f a}=9 \%$ & $p_{f a}=40 \%$ & $p_{f a}=21 \%$ & $p_{f a}=19 \%$ \\
\hline \multirow{2}{*}{ MRC with 2 antennas } & $p_{d}=34 \%$ & $p_{d}=45 \%$ & $p_{d}=58 \%$ & $p_{d}=57 \%$ & $p_{d}=60 \%$ & $p_{d}=80 \%$ \\
\hline & $p_{f a}=6 \%$ & $p_{f a}=40 \%$ & $p_{f a}=8 \%$ & $p_{f a}=22 \%$ & $p_{f a}=16 \%$ & $p_{f a}=10 \%$ \\
\hline \multirow{2}{*}{ MRC with 4 antennas } & $p_{d}=78 \%$ & $p_{d}=70 \%$ & $p_{d}=64 \%$ & $p_{d}=58 \%$ & $p_{d}=80 \%$ & $p_{d}=81 \%$ \\
\hline & $p_{f a}=16 \%$ & $p_{f a}=13 \%$ & $p_{f a}=8 \%$ & $p_{f a}=18 \%$ & $p_{f a}=15 \%$ & $p_{f a}=9 \%$ \\
\hline \multirow{2}{*}{ Collaborative with MRC } & $p_{d}=99 \%$ & $p_{d}=98 \%$ & $p_{d}=97 \%$ & $p_{d}=80 \%$ & $p_{d}=99 \%$ & $p_{d}=99 \%$ \\
\hline & $p_{f a}=28 \%$ & $p_{f a}=33 \%$ & $p_{f a}=18 \%$ & $p_{f a}=25 \%$ & $p_{f a}=21 \%$ & $p_{f a}=11 \%$ \\
\hline
\end{tabular}

From these two figures, it observed that Normal fading channel

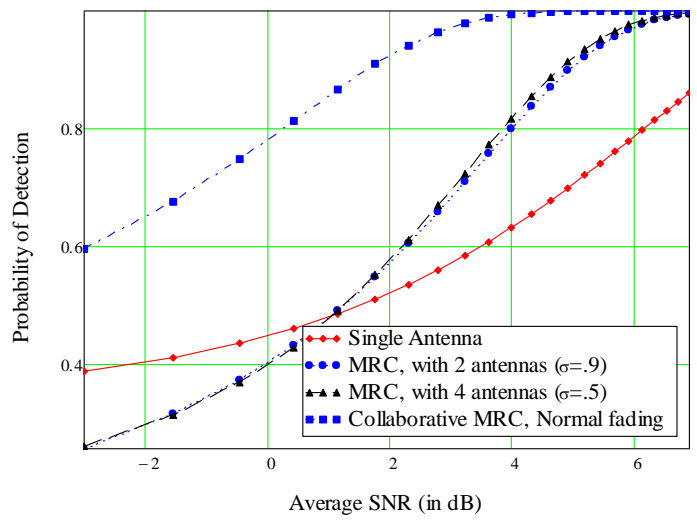

(a) Detection Probability under Normal fading

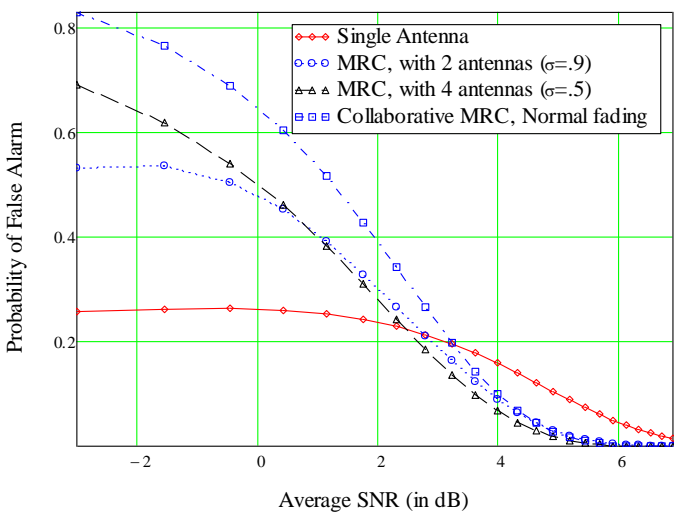

(b) False alarm probability under Normal fading

Fig. 12. Complementary ROC curves for detection and false alarm probability under Normal fading channel using Energy detection

In Figure 8, the effects of MRC under Rayleigh fading are studied using Energy detection. It is observed that collaborative MRC produces more effective result for higher SNR range.

Figures 9 and 10 show a clear comparison of the performance of detection between Bayesian and Energy detection under Nakagami- $m$ fading. These show that Nakagami- $m$ fading gives better detection than Rayleigh fading for same estimation.

Figures 11 and 12 manifests the ROC curves for Normal fading under Bayesian and Energy detection, which shows the effects of increasing number of antennas with MRC to estimate optimum detection by considering sensitive false alarm rate. start detection for low SNR in BD and produces better access probability for collaborative MRC where ED starts working at high SNR, and produces efficient detection against sensitive false alarm in collaborative environment.

In Table 4, relative performance of Nakagami- $m$, Rayleigh and Normal or Gaussian fading are given for different SNR level using $\mathrm{BD}$ and ED. Findings from this tables areCollaborative MRC produces a better output of access probability for all channels, Bayesian Detection works better at Nakagami-m fading for Low SNR values and Energy detection works better at Rayleigh fading for High SNR values.

\section{CONCLUSION}

This work provides the analysis of spectrum sensing using traditional Bayesian detection and Energy detection with MRC under Nakagami- $m$, Rayleigh and Normal fading channels. We have considered the collaborative spectrum sensing environment to maximise the access analysis for CRN user. Sample numbers and threshold identifications are very important for this proposed method due to dynamic changing environment which helps to improved CR performance. This work introduces an adaptive algorithm to conduct the spectrum sensing for hidden terminals that optimise the correct detection probability for collaborative CRN. From the simulated ROC curves, it is estimated that large number of samples make better performance and provide less misdetection. It is also observed that for large number of antennas MRC produce more correct decision with collaborative environment then local sensing under different fading channels. By analysis the relative performance of different wireless channel for various SNR levels it is showed that ED works better in high SNR and BD can works for low SNR.

\section{REFERENCES}

[1] K.Yau, P. Komisarczuk and P.D. Teal, "Cognitive Radio-Based Wireless Sensor Networks: Conceptual Design and Open Issues", IEEE $34^{\text {th }}$ Conference on Local Computer Network, pp. 955-962, 2009.

[2] S haykin, "Cognitive radio: Brain-empowered wireless communication", IEEE Journal Selected Areas in Communications, vol.23, no.2, pp.201-202, Feb.2005.

[3] Tevfik Yucek and Huseyin Arslan, "A survay of Spectrum Sensing Algorithms for Cognitive Radio Application" IEEE Communications and Tutorials, Vol. 11, No. 1, 2009.

[4] Aamir Zeb Shaikh, Dr. Talat Altaf, "Collaborative spectrum sensing under suburban environments", (IJACSA) International Journal of Advanced Computer Science and Applications, Vol. 4, No.7, 2013. 
[5] C. Sun, W.Zhang, and K. B. Letaief, "Cluster based cooperative spectrum sensing for cognitive radio system", in proc IEEE Int. Conf. Commun. Glasgow, Scotland, UK, pp. 2511-2515, June-2007.

[6] jianqi Lua, Ping Wei, "Optimization of Spectrum sensing over imperfect reporting channel", International workshop on Information and electronics engineering, 2012.

[7] Adeel Ahmed, Yim Fun Yu,James M.Nora , "Noise Variance estimationfor spectrum sensing in cognitive radio" AASRI Conference on circuitsand signal processing, 2014.

[8] Deepa Bhargavi and Chandra R. Murthy, "Performance Comparison of Energy, Matched-Filter and Cyclostationarity-Based Spectrum Sensing" IEEE Conference, July-2010.

[9] Mr. Pradeep Kumar Verma, Mr. sachin Taluja and Prof. Rajeshwar Lal Dua, "Performance analysis of Energy detection, Matched filter detection and Cyclostationary feature detection Spectrum Sensing Techniques", International Journal of Computational Engineering Research, pp.1296, Vol. 2, Issue. 5, September-2012.

[10] R. Vadivelu, K.Sankaranarayanan and V. Vijay akumari, “ Matched filter bades spectrum sensing for Cognitive Radio at low signal to noise ratio", Journal of theoretical and Applied Information Technology, Vol. 62, No. 1, 10 $0^{\text {th }}$ April, 2014.

[11] P.D. Sutton, K.E. Nolan, and L.E. Doyle, "Cyclostationary signatures in practical cognitive radio applications", IEEE J.Sel. Areas Commun., Vol. 26, No. 1, pp. 13-24, Jun-2008.

[12] F. F. Digham, M. S. Alouini, and M. K. Simon, "On the energy detectionof unknown signals over fading channels", IEEE Trans. Commun., vol.55, no. 1, pp. 2124, Jan. 2007.

[13] Padma Sai Prudvi, Lingaiah Jada and M. Siva Kumar, "Detection of Primary User in Cognitive Radio using Bayesian Approach" International Research Journal of Engineering and Technology (IRJET), Vol. 2, Issue. 5, Aug-2015.

[14] Aamir Zeb Shaikh, Dr. Talat Altaf, "Collaborative spectrum sensing under suburban environments", (IJACSA) International Journal of Advanced Computer Science and Applications, Vol. 4, No.7, 2013.

[15] W.Zhang and K.B Letaief, "Cooperative communications for cognitive radio networks", Proc. IEEE, Vol.97, No. 5, pp. 805-823, May 2009.

[16] S. M. Mishra , A. Sahai and R.W. Brodersen, "Cooperative sensing among cognetive radios", in Proc. IEEE Conf. Commun., pp. 16581663, June 2006.
[17] J. Shen, T.Jiang, S.Liu and Z.Zhang, "Maximum channel troughput via cooperative spectrum sensing in cignitive radio networks", IEEE Trans. Wireless Commun., Vol.7, No. 10, pp. 5166-5175, Oct., 2009.

[18] D.cabric, S.Mishra, R.Brodersen , "Implementation issues in spectrum sensing for cognitive radios" in: Proc. Of Asilomar Conf. on Signals, System and Computers, vol.1, pp.772-777, 2004.

[19] D. Teguig , B. Scheers and V. Le Nir, "Data fusion schemes for cooperative spectrum sensing in cognitive radio networks", Communicationsand Information Systems Conference (MCC), Military, IEEE 8-9 , pp:1 7 Print ISBN: 978-1-4673-1422-0, Oct.2012.

[20] F. F. Digham, M. S. Alouini, and M. K. Simon, "On the energy detectionof unknown signals over fading channels", IEEE Trans. Commun., vol.55, no. 1, pp. 2124, Jan. 2007.

[21] Risala Tasin Khan, Shakila Zaman, Md. Imdadul Islam and M. R. Amin, "Optimum Access Analysis of Collaborative Spectrum Sensing in Cognitive Radio Network using MRC", (IJACSA) International Journal of Advanced Computer Science and Applications, Vol. 7, No.7, 2016.

[22] F. F. Digham, M. S. Alouini and M. K. Simon, "On the energy detection of unknown signals over fading channels", in Proc IEEE ICC, pp. 35753579, May 2003.

[23] Hongjian Sun, David. Laurenson and Chengxiang Wang, "Computationally Tractable mode of energy detection performance over slow fading channels", IEEE Commun., Vol. 14, Issue. 10, Oct. 2010.

[24] E. Visotsky, et al., "On collaborative detection of TV transmissions in support of dynamic spectrum sharing", in New Frontiers in Dynamic Spectrum Access Networks, First IEEE International Symposium on, pp.338-345, 2005.

[25] Deep Raman and N.P.Sing , "An Algorithm for spectrum sensing in Cognitive Radio under noise uncertainty", International journal of Future Generation communication and Networking, Vol.7, No. 3, pp. 61-68, 2014.

[26] Erik Axell, Geert Leus, Erik G. Larsson and H. Vincent Poor, "State-ofthe-art and recent advances Spectrum Sensing for Cognitive Radio State-of-the-art and recent advances", IEEE signal processing magazine , Vol. 29, Issuse. 3, pp. 101-116, 2012.

[27] Simon O. Haykin, Michael Moher, "Modern Wireless Communications", ISBN-10: 0130224723. 